\title{
Delay in Treatment of Testicular Tumours in the Army
}

\author{
Major G J Attard, \\ $M D, F R C S(E d)$, RAMC \\ Queen Elizabeth Military Hospital, Woolwich
}

SUMMARY: Modern drug treatment, added to our previous experience of surgery and radiotherapy, is currentlyœ producing nearly $90 \%$ cure of all patients with testicular tumours. Most of the failures of treatment today occur in $\vec{\circ}$ patients with a large mass of diseased tissue which has accumulated due to delayed diagnosis. A retrospective study of 23. Servicemen with testicular tumours, treated at the QEMH Woolwich, shows that the blame for this delay is shared by the $\vec{\omega}$ patient, his medical practitioner, and surprisingly enough the hospital specialist. Recommendations for improvement are discussed.

\section{Introduction}

The incidence of testicular tumours has been steadily increasing since the earlier part of the century. They are now the most frequent malignancy in males between the late teens and forty years. ${ }^{1}$. About eight new cases are referred each year to the Queen Elizabeth Military Hospital in Woolwich.

With the introduction of effective chemotherapy the prospects for cure approach one hundred per cent for early disease and, even for very bulky metastatic disease, cure rates of sixty per cent are obtained ${ }^{2}$. It is therefore imperative that aggressive management, with early diagnosis and rapid treatment, is instituted, particuarly in those patients with atypical presentation, who have the worst long term prognosis.

Testicular tumours are notorious for the delay in which they are treated. This is partly due to the patient's own delay in presenting himself to a doctor and, not uncommonly, further compounded by a misdiagnosis.

\section{Method}

A retrospective study of twenty-three patients with testicular tumours, who attended the Queen Elizabeth Military Hospital for treatment or follow-up in the Neoplastic Clinic, was carried out. Cases of delay in presentation and misdiagnosis were carefully analysed to see if improvement could be made to current management.

\section{Results}

Fourteen cases were diagnosed as teratomas, eight as seminomas, and one case was a mixed germ cell tumour.

In only one case was there a history of testicular maldescent, the patient having had an ipsilateral orchidopexy in early childhood. None of the patients gave a history of mumps orchitis.

In fifteen cases there was a delay of more than two months in starting treatment. The period varied from
Table 1

Causes of delay in starting treatment

(a) Failure of patient to seek medical advice - 6

(b) Misdiagnosis by patient's medical officer -11

(c) Misdiagnosis by hospital specialist -6

Table 2

Reasons given by patients for not seeking early medical advice

Did not think swelling was important -3

Attributed swelling to trauma -2

Not aware of any swelling -1

three months to as long as three years. Table 1 shows th causes of delay in initiating treatment.

Six cases of delay were partly due to the patient failing to seek early medical advice. Table 2 shows the reasons? given by patients not seeking early medical advice.? Three of the patients believed the swelling was unimportant as it was painless. One patient was aware of a progressive, painless swelling in his testis for three years. He finally consulted his doctor after he developed abdominal pain from para-aortic node metastasis. In two patients the testicular swelling was first noticed after? a kick in the scrotum and then wrongly attributed by patient and doctor alike to trauma. One patient with a large obvious swelling was unaware of its presence until it was pointed out at a routine medical.

In eleven cases there was a delay in hospital referral due to misdiagnosis by the patient's medical officer. The $>$ commonest difficulty was confusion with epididymoorchitis. Unfortunately in two cases the doctor did not follow the patient up. In a third case a further course of antibiotics was given when the swelling failed to resolve, thereby causing an unnecessary delay. Three cases were diagnosed as renal colic and back pain.

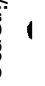


Six patients were misdiagnosed by the hospital specialist also at the first examination causing an unnecessary delay. It was evident that the correct diagnosis could have been reached in all but two cases, had a complete clinical examination of the patient, a routine chest $X$-ray, and testicular tumour markers been carried out when the patient was first seen. In the other two cases the diagnosis could only have been made by a surgical exploration. Unfortunately in one case there was unnecessary delay in exploration as a result of adopting a prolonged expectant policy in a case of 'epididymo-orchitis'.

Table 3 shows the histological classification, the medical officer's diagnosis and initial hospital diagnosis in eleven cases showing a delay in starting treatment.

Table 4 gives the staging at orchidectomy in misdiagnosed cases.

Table 3

Histological classification and original diagnosis in misdiagnosed cases

\begin{tabular}{lll}
\hline Histology & MO's diagnosis & Specialist's diagnosis \\
\hline MTU & Epididymo-orchitis & Epididymo-orchitis \\
MTU & Epididymo-orchitis & Neoplasm \\
MTD & Epididymo-orchitis & Epididymo-orchitis \\
MTI & Epididymo-orchitis & Torsion \\
Seminoma & Epididymo-orchitis & Neoplasm \\
MTU & Haematocele & Haematocele \\
MTD & Idiopathic & Idiopathic \\
& Gynaecomastia & Gynaecomastia \\
MTD & Loin Pain & Neoplasm \\
MTI & Renal Colic & Neoplasm \\
Seminoma & Back Pain & Neoplasm \\
Mixed Germ & Patient not & Diagnosis made at \\
Cell & examined & follow up after a \\
& & vasectomy! \\
\hline
\end{tabular}

\section{Discussion}

Most of the failures in treatment today occur in patients with a large mass of disease which has accumulated due to delayed treatment. New drugs and combination therapies are being developed with the aim of increasing the cure rate of this difficult group. A concerted effort to educate servicemen to seek medical advice earlier, and a higher index of suspicion among doctors, at all levels of management, is likely to be more rewarding at present.

Despite the serious nature of lumps in the testis having been known since Roman times, this knowledge is not at all widespread among the male population. Men have not been trained as effectively as women to look after their sexual apparatus. Certainly, soldiers can be instructed to report lumps in their testes as they would a urethral discharge. Rank does not appear to have any privileges as a fairly senior officer was aware of a painless swelling in his testis for over a year, but did nothing
Table 4

Staging at orchidectomy in misdiagnosed cases

SEMINOMA

$$
\begin{aligned}
& \text { Stage } 1 a-1 \\
& \text { Stage } 4-1
\end{aligned}
$$

TERATOMA

$$
\begin{aligned}
& \text { Stage 1a-3 } \\
& \text { Stage } 2-1 \\
& \text { Stage } 3-2 \\
& \text { Stage } 4-3
\end{aligned}
$$

about it. It was detected at routine follow up two weeks after a vasectomy!

Routine examination of the testis of every soldier who attends sick parade would be unrealistic. However routine examination of the genitalia should be carried out in every case of back pain, abdominal pain, or renal colic seen. Three cases were misdiagnosed simply because the testes were never examined.

The patient who attends with gynaecomastia - be ito unilateral or bilateral - should similarly have his testis examined and then referred for a specialist opinion.

There is much to be said for the suggestion of 're- education of military doctors' in order to dispel the $\frac{\vec{P}}{\mathrm{D}}$ classical textbook description of a painless swelling that tends to dominate the thinking of quite a few of us ${ }^{3}$. The commonest cause of confusion is with inflammatory disease and trauma: indeed, a testis containing tumour $\varnothing$ may get smaller with antibiotics ${ }^{2}$. However there is noo or justification for prolonged follow up of an epididymoorchitis that fails to resolve, or for prescribing an antibiotic and not seeing the patient again.

An epididymo-orchitis that has failed to resolve after a month following a standard course of antibiotics warrants urgent hospital referral. If there is still serious doubt a surgical exploration is indicated, and not a further course of antibiotics and procrastination.

Similarly a haematocele that fails to resolve rapidly should be viewed with suspicion and explored.

Finally all patients with suspicious testicular swellings should be treated as surgical emergencies, and referred as such to hospital.

\section{Acknowledgement}

I wish to thank Colonel N. Peters L/RAMC for permission to report on his cases.

\section{REFERENCES}

1. Oliver R T D. Progress in the management of testicular germ-cell tumours, Practitioner 1982; 226: 1903-1915.

2. BarRet A. Testicular Tumours, Hospital Update 1984; 10: $355-369$.

3. JONES R T S. Giants Cell Tumours of the testis, Update 1984; 28 : 1147-1154. 